\title{
CYANOPHYCEAE/CYANOBACTERIA IN RED MANGROVE FOREST AT MOSQUITOS AND COQUEIROS ESTUARIES, SÃO LUÍS, STATE OF MARANHÃO, BRAZIL
}

\author{
NOGUEIRA, N. M. C. and FERREIRA-CORREIA, M. M. \\ Departamento de Oceanografia e Limnologia da Universidade Federal do Maranhão, \\ Praça Gonçalves Dias, 21, CEP 65020-240, São Luís, MA, Brazil \\ Correspondence to: Naiza Maria Castro Nogueira, Rua Miragem do Sol, 15, Ed. Torricelli, ap. 801, \\ Renascença II, CEP 65075-760, São Luís, MA, Brazil, e-mail: nangueira@elo.com.br \\ Received June 16, 1999 - Accepted July 19, 2000 — Distributed August 31, 2001
}

(With 36 figures)

\begin{abstract}
This paper provides the results of a taxonomic survey of the Cyanophyceae/Cyanobacteria in a frenge red mangrove forest in the estuaries of Estreito dos Mosquitos and Coqueiros, São Luís, State of Maranhão, Brazil. A total of 15 taxa were identified in 8 families, as follows: Synechoccaceae (2), Chroococcaceae (1), Hyellaceae (1), Xenococcaceae (1), Oscillatoriaceae (1), Scytonemataceae (2), Phormidiaceae (5) and Pseudanabaenaceae (2). The species listed in this paper are all new descriptions for Maranhão, and one of them is a new ocurrence for Brazil.
\end{abstract}

Key words: mangrove Cyanobacteria, estuaries, Rhizophora forest.

\section{RESUMO}

Cyanophyceae/Cyanobacteria em um bosque de mangue vermelho nos estuários dos Estreitos dos Mosquitos e dos Coqueiros, São Luís, Estado do Maranhão, Brasil

Este trabalho fornece resultados de um levantamento taxonômico das Cyanophyceae/Cyanobacteria em um bosque de franja de mangue vermelho nos estuários dos Estreitos dos Coqueiros e dos Mosquitos, São Luís, Estado do Maranhão, Brasil. Um total de 15 táxons foram identificados em 8 famílias, como segue: Synechoccaceae (2), Chroococcaceae (1), Hyellaceae (1), Xenococcaceae (1), Oscillatoriaceae (1), Scytonemataceae (2), Phormidiaceae (5) e Pseudanabaenaceae (2). Todas as espécies listadas neste artigo são descrições novas para o Maranhão e uma delas é citação nova para o Brasil.

Palavras-chave: Cyanobacteria de manguezal, estuários, bosque de Rhizophora.

\section{INTRODUCTION}

According to Dor (1984), the main focus of papers on mangrove associated algae has been on "Bostrychietum", the species communities belonging to the genera Bostrychia, Catenella and Caloglossa. It is interesting to notice that studies of blue-green mangrove algae are usually secondary objectives on mangrove algae research worldwide (Branco, 1991).

Taxonomic studies on the Cyanophyceae are very scarce and limited to Dor (1984), who studied the vertical zonation and morphological adaptations of the epiphytic blue-green algae found at Sinai Estuary's mangroves and Lambert et al. (1989), who reported 27 taxa of blue-green algae and their relative abundance (in different substracts), for South African mangroves.

In the case of Brazil, there are four papers that focus specifically on the cyanophytes from mangroves: Branco (1991), Neves \& Tribuzi (1992), Branco et al. (1996) and Branco et al. (1997). All papers of Branco (1991) and Branco et al. $(1996,1997)$ compiled the cyanophyte flora from Ilha do Cardoso and Neves \& Tribuzi (1992) studied mangrove cyanophyte from "Ponta do Pai Vitório", Cabo Frio, Rio de Janeiro State. Another references are in: Möebius (1889), who reported the ocurrence of Microcoleus chtonoplastes Thur. ex Gom. in Joinville's mangroves; Joly (1951) listed 
Lyngbya confervoides C. Ag. ex Gom. Dermocarpa sp., Sirocoleum sp. and Stigonema sp., in Paraná State mangroves; Joly (1957) reported the presence of Sirocoleum guyanense Kütz. ex Gom. in Santos and surrounding mangroves; Sant'Anna et al. (1983) found Oscillatoria bornetti Zukal and $O$. princeps Vauch. ex Gom. in the Ilha do Cardoso mangroves; Oliveira (1984) observed the genera Oscillatoria, Lyngbya, Microcoleus and Scytonema in the Brazilian mangroves; Carmo (1987) cited Microcoleus vaginatis (Vauch.) Gom. ex Gom. as a representative species for the blue-green algae in the mangroves located in the North of Baía de Vitória, Espírito Santo State; Sant'Anna (1988) described a new species, Scytonema insularis, from the Ilha do Cardoso mangroves; and Moura (1991) recorded the occurrence of 29 Cyanophyceae taxa in a quali-quantitative study of periphytic algae in the rivers Paripe and Igarassu-Itamaracá Estuaries, in Pernambuco State.

In Maranhão, mangrove ecosystems have been studied by Frois-Abreu (1939), Damázio (1979/1980a, 1979/1980b), Damázio \& Santos (1985, 1990), Santos \& Damázio (1985), Santos (1986, 1989, 1997), and Mochel et al. (in press). Components of phycological associated flora are found in Ferreira-Correia \& Brandão (1974), Ferreira-Correia et al. (1977), Ferreira-Correia (1986) and Cutrim (1998).

There is a total lack of knowledge on the phycological flora in frenge red mangrove forest. Regarding the taxonomy of the Cyanophyceae, no studies on estuarine mangrove environments, such as those of Maranhão, have been published.

This paper provides the results obtained from a qualitative study of the Cyanophyceae growing on Rhizophora dominated mangroves at Mosquitos and Coqueiros estuaries, in the Maranhão State, Brazil.

\section{MATERIAL AND METHODS}

The Mosquitos strait separates São Luís Island from the continent. This strait is $5 \mathrm{~km}$ long and $104 \mathrm{~m}$ wide, extending from Southeast to Northwest, and linking the Arraial Bay with São Marcos Bay. The coordinates are latitude 2'38'12'"S and longitude 4423'35' W (Fig. 1).

Two sampling areas were chosen, and in each one, a vertical transect (seaward landward) was established. Along the transects, stations were marked at $100 \mathrm{~m}$ intervals, and samples collected every $10 \mathrm{~m}$. Algae were collected by scrapping the trunks and roots of Rhizophora mangle L, where they usually grow.

In each area, five points were taken, and 12 samples were made from each one, totaling 60 samples from the Mosquitos strait, and also 60 samples from the Coqueiros strait.

Samples were stored in polietilene bottles and preserved in $4 \%$ formaldehyde solution. They were finally deposited in the Biology Herbarium (HDB) of the Federal University of Maranhão.

The distribution of the species in Brazil, is presented by states.

The systems of classification used in this study were those by Anagnostidis \& Komárek (1988) for Oscillatoriales, Komárek \& Anagnostidis (1989) for Nostocales, and Komárek \& Anagnostidis (1999) for Chroococcales.

\section{RESULTS}

The Cyanophyceae estuarine flora consisted of the taxa described as follow:

\section{Chroococcales}

Synechococcaceae

Aphanothecoideae

Aphanothece Näg., 1849

A. cf. castagnei (Bréb.) Rabh.

Flor. Eur. Alg., II: 64, 1865.

Basionym: Palmella castagnei Kütz., Tab. Phyc., 1:9, 1846. (Fig. 2)

Colonies amorphous, mucilaginous, limous, blue-green or dark, with more or less densely and irregularly arranged cells, near the colonial margin regularly surrounded by their own, usually slightly lamellate and yellowish brown envelopes; cells oval or cylindrical, with widely rounded ends, 4$6.3 \times 2.3-3.6 \mu \mathrm{m}$.

Material examined: Mosquitos and Coqueiros estuaries. Mosquitos Estuary: HDB1540, 1543, 1553 and 1557; Coqueiros Estuary: HDB1567, 1587 and 1612 .

Distribution in Brazil: Distrito Federal (Senna, 1992); Pernambuco (Mora, 1990), Rio de Janeiro (Neves, 1988, 1991); Rio Grande do Sul (Franceschini, 1991); São Paulo (Sant'Anna et al., 1991; Branco et al., 1996).

Comments: Acording Komárek \& Anagnostidis (1999), Aphanothece castagnei does not occur in aquatic biotops; this statement has made the 
accurate identification of the species more difficult. However, the material studied showed the same habitat conditions as described by Branco et al. (1996), in Rio de Janeiro and São Paulo States: plant massed formed on the mud accumulated on plantlets.

A. cf. stagnina (Sprengel) A. Braun.

Rabenh., Flor. Eur. Alg., 2:66, 1863. (Figs. 5 and 6)

Colonies macroscopic, gelatinous, with distinct, firmly delimited margin, spherical or irregularly elongate and lobate, usually consisting of subcolonies, to $1.0 \mathrm{~cm}$ in diameter; sheath bluegreen or dark homogeneous, with inner calcareous cristals; cells oval to widely cilindrical, 4.6-6.1 $\times$ 2.2-2.7 $\mu \mathrm{m}$, dense or loosely dispersed cells in the surroundings of the colony, and loosely dispersed cells in the center of colony.

Material examined: Mosquitos Estuary: HDB1548, 1562; Coqueiros Estuary: HDB1565, 1575, 1601 and 1613 .

Distribution in Brazil: Distrito Federal (Cronberg, 1978), Minas Gerais (Sant'Anna, 1984); Rio de Janeiro (Neves, 1983, 1990); Rio Grande do Sul (Coutinho, 1982; Werner, 1984; Franceschini, 1991; Werner \& Rosa, 1992).

Comments: Material perifytic, living on mangrove macroalgae. Komárek \& Anagnostidis (1999) stated that material from marine water or mangrove are questionable and need confirmation.

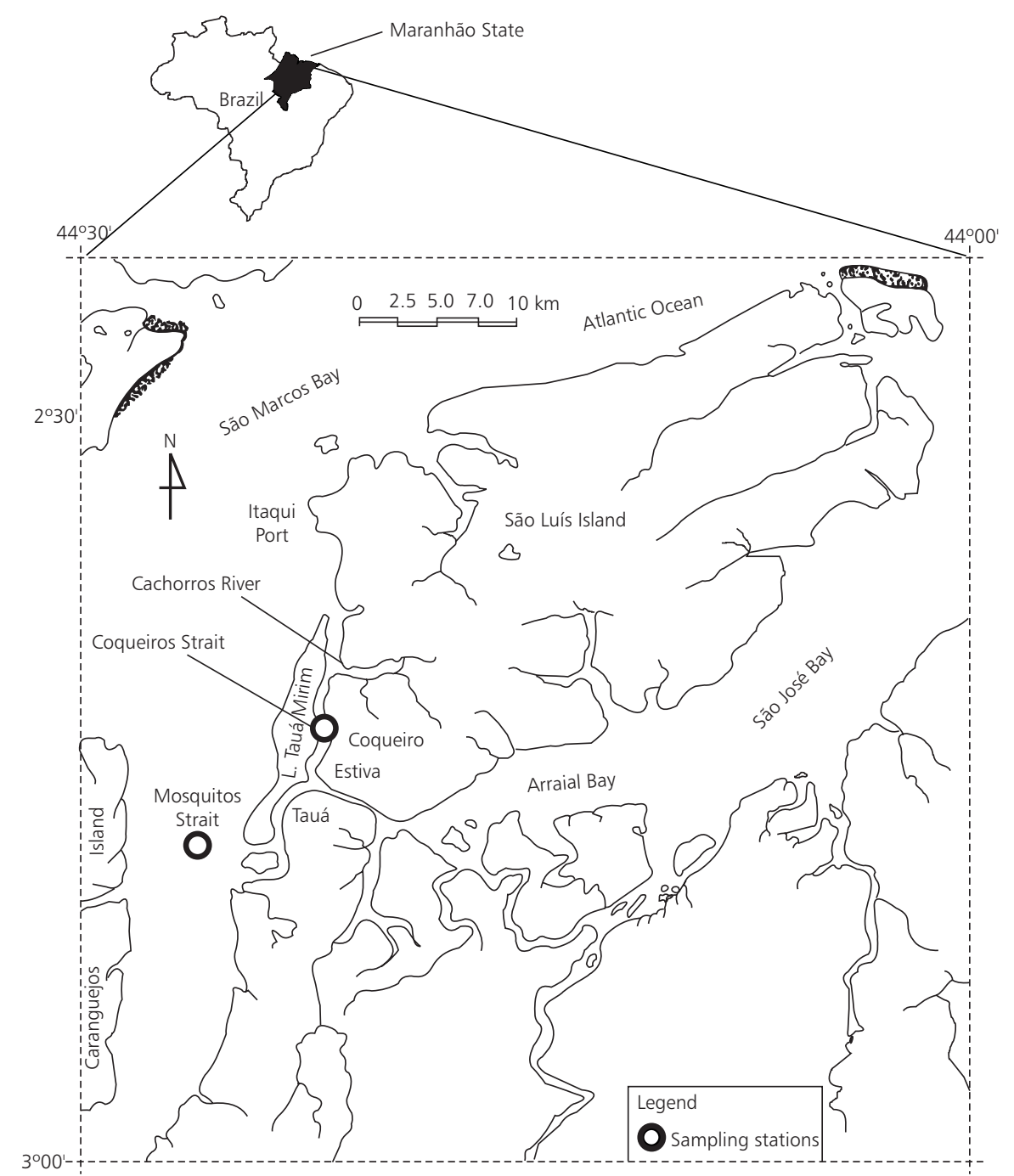

Fig. 1 - Location of the sampling stations. 
Because of this, the specimen studied could not be acurately identified.

Chroococcaceae

Gloeocapsopsis Geit. ex Kom. 1993.

G. crepidinum (Thur.) Geit. ex Kom. 1993. Basionym: Gloeocapsa crepidinum Thur. (Figs. 3 and 4)

Colonies formed by cells in 2-4 set groupings, round or ovoid, 12.0-14.0 $\mu \mathrm{m}$ diameter; sheath yellow or dark in the outer part and colourless or difluent inside, without lamellas; cells round rare but thickly arranged in the peripheric region and spread out at the centre, 1.8-4 $\mu \mathrm{m}$ diameter.

Material examined: Mosquitos Estuary: HDB1539, 1545 and 1561.

Distribution in Brazil: Rio de Janeiro (Neves, 1988, 1991); Rio Grande do Sul (Coutinho, 1982); São Paulo (Sant'Anna, 1995; Sant'Anna et al., 1995).

Hyellaceae

Hyelloideae

Pleurocapsa sp. (Fig. 8)

Almost gelatinous colonies of varied size and forms; mutually pressed spherical or angular cells; blue-greenish, olive green, yellow or violet cellular content.

Material examined: Mosquitos Estuary: HDB1537, 1555; Coqueiros Estuary: HDB1572, 1580, 1585, 1597 and 1608.

Comments: The material was not identified at the specific level because it was insufficient, so it was impossible to measure it.

Xenococcaceae

Xenococcus Thur. in Born. et Thur. 1880.

X. pyriformis Setch. et Gardn. in Gardn. 1918. University of California Publications in Botany, 6:436, 1918. (Figs. 7 and 9)

Colonies small, shining, blue-green, singular or occasionally coalescent, 9.0-11.0 $\mu \mathrm{m}$ long, $5.4 \mu \mathrm{m}$ diameter; sheath conspicuous, dense, hyaline; cells slight angular when young, and pyriforms subsphericals when adult; same bacocyte angle and size for all cells, gonads formed by successive divisions.

Material examined: Mosquitos Estuary: HDB1536, 1550 and 1558; Coqueiros Estuary: HDB1566, 1576, 1590, 1603 and 1609.

Distribution in Brazil: São Paulo (Sant'Anna et al., 1985).
Scytonemataceae

Scytonema Born. et Flah. ex C. Ag. 1887.

S. arcangeli Born. et Flah.

Ann. Sci. Nat. series Bot., 7(5):92, 1887. (Figs. 10, 11 and 13)

Plant mass blue green; filaments loosely entangled, 14.9-18.3 $\mu \mathrm{m}$ broad; false branches in pairs and single, frequent; sheath homogeneous or with parallel layers, colourless to brownish; some parts of the trichomes constricted; 9.5$15.0 \mu \mathrm{m}$ broad; cells 5.3-8.0 $\mu \mathrm{m}$ long, contents blue-green; heterocytes intercalary, round or squarish, 6.8-10.9 $\mu \mathrm{m}$ long, 7.4-10.0 $\mu \mathrm{m}$ broad.

Material examined: Mosquitos Estuary: HDB1534 and 1535; Coqueiros Estuary: HDB1568, 1579, 1593, 1596 and 1607.

Distribution in Brazil: São Paulo (Sant'Anna, 1988; Branco, 1991).

\section{S. insulare Sant' Anna.}

Nova Hedwigia, 46(3-4):528, 1988. (Figs. 12, 14 and 15)

Plant mass blue-green; filaments tightly entangled, 9.7-12.0 $\mu \mathrm{m}$ broad; false branches in pairs, parallel, frequent; sheath firm, homogeneous; trichomes generally constricted and slightly atenuated at the apex, cells 4.0-7.8 $\mu \mathrm{m}$ long, 5.2-6.2 $\mu \mathrm{m}$ broad; heterocytes intercalary, squarish, cylindrical or sometimes rounded, 4.0-8.0 $\mu \mathrm{m}$ long, 4.0-7.6 $\mu \mathrm{m}$ broad.

Material examined: Mosquitos Estuary: HDB1534, 1546 and 1554; Coqueiros Estuary: HDB1556, 1563, 1574, 1584, 1594 and 1605.

Distribution in Brazil: São Paulo (Sant'Anna, 1988; Branco, 1991).

Oscillatoriales

Phormidiaceae

Porphyrosiphon Kütz. ex Gom. 1892.

P. luteus (Gom.) Anagn. et Kom.

Basionym: Lyngbya lutea Gom.

Ann. Sci. Nat., 16:141, 1892. (Figs. 18 and 19)

Filaments solitary or entangled 5.3-7.0 $\mu \mathrm{m}$ broad, above $3 \mu \mathrm{m}$ long; sheath hyaline, homogeneous; trichomes straight, not attenuated, not constricted, 4.0-4.2 $\mu \mathrm{m}$ broad; cells 1.2-1.3 times shorter than broad, 3.1-4.7 $\mu \mathrm{m}$ long; cross walls not granulated; cell content blue-green, homogeneous; apical cell rounded-conical, with or without thickened outer membrane.

Material examined: Coqueiros Estuary. 


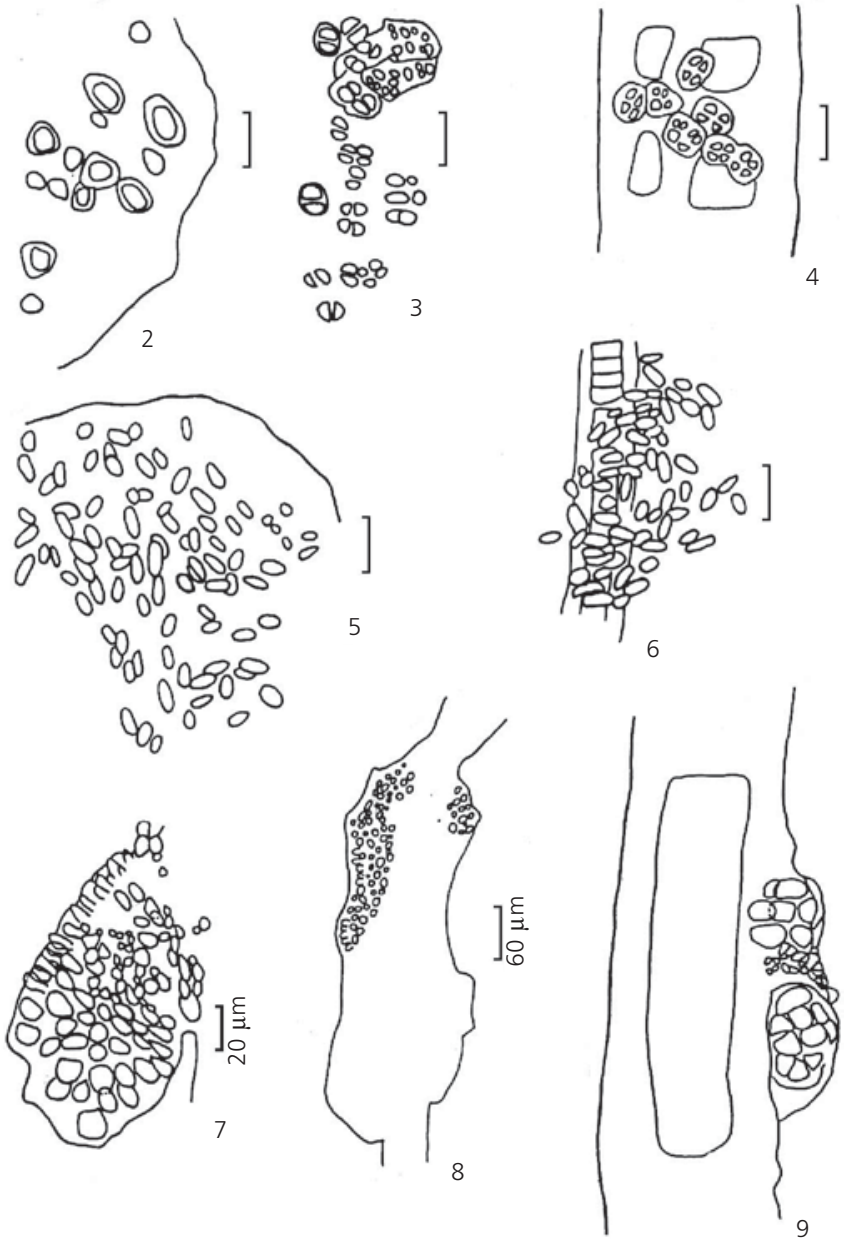

Fig. 2 - Aphanothece castagnei (Bréb.) Rabh. Figs. 3-4 - Gloeocapsopsis crepidinum (Thur.) Geit. ex Kom. Figs. 56- Aphanothece cf. stagnina (Spreng) A. Braun - colony growing on B. calliptera (Montagne) Montagne. Fig. 7 and 9 - Xenoccocus pyriformes Setchell et Gardner. Fig. 9 - growing on B. calliptera (Montagne) Montagne. Fig. 8 Pleurocapsa sp. (Figs. 2-6: scale bars represent $10 \mu \mathrm{m}$; Figs. 8-9: scale bars represent $60 \mu \mathrm{m}$ ).

Distribution in Brazil: Rio de Janeiro (Neves, 1988); Rio Grande do Sul (Coutinho, 1982); São Paulo (Sant'Anna et al., 1985; Branco et al., 1997; Sant'Anna \& Simonetti, 1992; Sant'Anna, 1995).

Comments: The material wasn't deposited in herbarium because it was insufficient.

\section{Oscillatoriaceae}

Lyngbya C. Ag. ex Gom. 1824.

L. aestuarii Lieb. ex Gom.

Ann. Sci. Nat., 16:127, 1892. (Figs. 20 and 21)

Mass slightly green; filaments caespitose, 17.4-30.0 $\mu \mathrm{m}$ broad; sheaths hialine, homogeneous or lamellated, 2.0-6.2 $\mu \mathrm{m}$ width; trichomes constricted, 8.8-15.7 $\mu \mathrm{m}$ broad; cells round, 4 times wider than long, 1.9-5.3 $\mu \mathrm{m}$ long; content bluegreen; cross walls granulated or not; apex usually not thickened.

Material examined: Mosquitos Estuary: HDB1533 and 1552; Coqueiros Estuary: HDB1578, 1589 and HDB1599.

Distribution in Brazil: São Paulo (Sant'Anna et al., 1985; Neves \& Tribuzi, 1992; Azevedo \& Sant'Anna, 1993).

Pseudanabaenaceae

Leptolyngbyoideae

Leibleinia (Gom.) L. Hoffm, 1985.

L. pellucida (Umezaki) Branco, Sant'Anna, Azevedo \& Sormus. 
Basionym: Lyngbya pellucida Umezaki. Bot. Mag., 68(801):68, 1955. (Figs. 16 and 17)

Filaments solitary, 2.5-3.0 $\mu \mathrm{m}$ broad; sheath hyaline, homogeneous; trichome straitght or curve, not attenuated, not constricted, 1.6-1.8 $\mu \mathrm{m}$ broad; cells 1.2 to 1.3 times longer than wider, $1.5-2.1 \mu \mathrm{m}$ long; cross walls not granulated, pellucid; cell content green, homogeneous; apical cell conical rounded, without thickened outer membrane.

Material examined: Mosquitos Estuary: HDB1541 and 1560; Coqueiros Estuary: HDB1577, 1588, 1604 and HDB1610.

Distribution in Brazil: São Paulo (Sant'Anna et al., 1985; Branco et al., 1997; Sant'Anna \& Simonetti, 1992).

Microcoleus Gom. ex Desma. 1892.

M. chthonoplastes Gom.

Ann. Sci. Nat., 15:253, 1892. (Figs. 22-24)

Filaments solitary or just a few together, 25.9-

$36.0 \mu \mathrm{m}$ broad; sheath broad, hyaline; trichomes constricted, not attenuated; cell content green, homogeneous; apical cell rounded-conical, without thickened outer membrane.

Material examined: Mosquitos Estuary: HDB1538, 1541 and 1560; Coqueiros Estuary: HDB1564, 1583, 1591 and 1606.

Distribution in Brazil: Rio de Janeiro (Neves, 1983, 1990, 1992; Neves \& Tribuzi, 1992); Rio Grande do Sul (Coutinho, 1982); Santa Catarina (Möebius, 1889; Drouet, 1937); São Paulo (Sant'Anna et al., 1985; Branco et al., 1997; Sant'Anna \& Simonetti, 1992).

Comments: The length and width of the trichomes were not measured because the material was insufficient.

\section{M. tenerrimus Gom.}

Ann. Sci. Nat., 15:355, 1892. (Figs. 25 and 26)

Filaments solitary or few together; sheath broad, hyaline; trichomes slightly constricted, not attenuated; cells 3 times longer than wider, 4.8$7.0 \times 1.6-2.6 \mu \mathrm{m}$; cell content blue-green to green, homogeneous; apical cell acicular, long, sometimes curved, without thickened outer membrane.

Material examined: Mosquitos Estuary: HDB1544; Coqueiros Estuary: HDB1571, 1582, 1592 and HDB1595.

Distribution in Brazil: Rio Grande do Sul (Coutinho, 1982); São Paulo (Branco et al., 1997).
Phormidium Kütz. ex Gom. 1892.

P. corallinae (Gom. ex Gom.) Anagn. et Kom. Basionym: Oscillatoria corallinae Gom.

Ann. Sci. Nat., 16:218, 1892. (Figs. 27-30)

Tricomes, isolated, not attenuated, 6.4-6.8 $\mu \mathrm{m}$ broad; cells 2-3 times longer than wider, 2.2$3.4 \mu \mathrm{m}$ long; blue-green content, cross walls finely granulated or not; apical cell rounded, slightly twisted; slender apex.

Material examined: Coqueiros Estuary (HDB1569).

Distribution in Brazil: Rio de Janeiro (Neves \& Tribuzi, 1992); São Paulo (Sant'Anna et al., 1985; Sant'Anna \& Simonetti, 1992).

Pseudanabaenaceae

Leptolyngbyoideae

Leptolyngbya Anagn. \& Kom. 1988.

L. cf. fragilis (Gom) Anagn. \& Kom.

Basionym: Phormidium fragile Gomont. (Figs. 3133)

Filaments muscilagenous, lamellated, yellowish or brownish blue-green; sheath diffluent; trichomes more or less flexuous, distinctly constricted, septa not granulated, attenuated at the ends, 3.4-4.0 $\mu \mathrm{m}$ broad; cells nearly quadrate, 2.5-3.0 $\mu \mathrm{m}$ long, end cell acute-conical, calyptra absent.

Material examined: Coqueiros Estuary.

Distribution in Brazil: Rio Grande do Sul (Coutinho, 1982).

Comments: This species was not identified with accuracy because of the diameter of trichomes. Our material presents trichomes with diameter higher than the studied by Desikachary (1959) but, it occur in the same habitat, estuary water.

The material was not deposited in the herbarium because it was insufficient.

L. crosbyana (Til.) Anagn. et Kom. Basionym: Phormidium crosbyanum Tilden. (Fig. 34)

Filaments solitary, 2.0-4.0 $\mu \mathrm{m}$ broad; sheath hialine, delicate, homogeneous; trichomes tarnished brown, not attenuated, not constricted, 1.9-2.5 $\mu \mathrm{m}$ broad; cells 1.9 times longer than wider, 3.6-7 $\mu \mathrm{m}$ long; apical cell conical-rounded, without calyptra.

Material examined: Coqueiros Estuary.

Distribution in Brazil: reported for the first time for Brazil.

Comments: The material was not deposited in the herbarium because it was insufficient. 


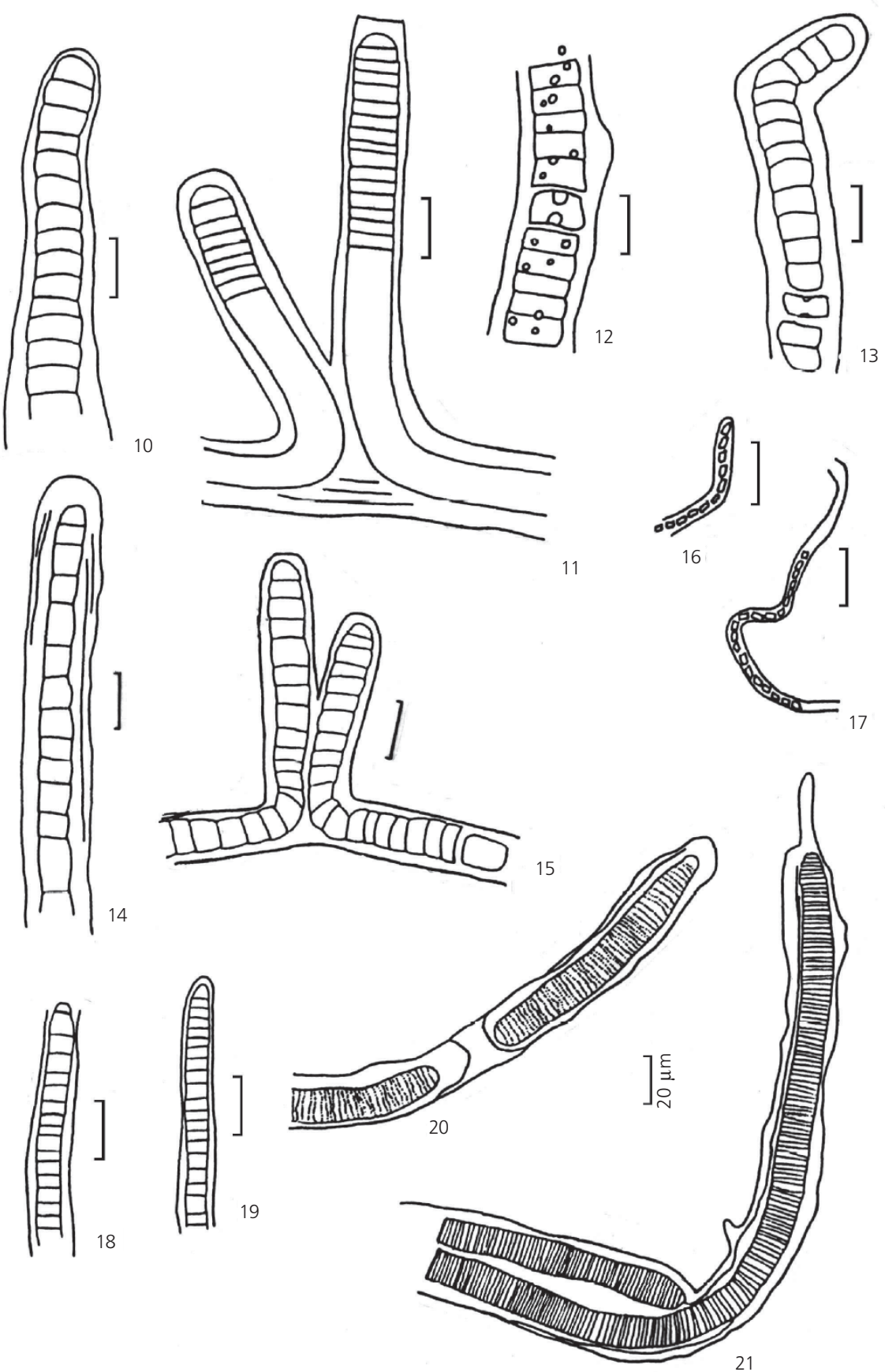

Figs. 10, 11 and $13-$ Scytonema arcangeli Bornet et Flahault. Fig. $10-$ Rounded apex; $11-$ False branched; $13-$ Trichome with intercalar heterocyte. Figs. 12, 14 and $\mathbf{1 5}$ - Scytonema insulare Sant'Anna. 12 - Trichome with intercalar, square heterocyte; 14 - Rounded apical cell; 15 - False branched. Figs. 16-17 - Leibleinia pellucida (Umezaki) Branco, Sant'Anna, Azevedo \& Sormus. Figs. 18-19 - Porphyrosiphon luteus (Gomont) Anagnostidis et Komárek. 18 - Apical cell conical; 19 - Apical cell rounded. Figs. 20-21 - Lyngbya aestuarii Liebmann ex Gomont. 20 Filament with hormogonio and granulated cross-wall; 21 - Filaments with two trichomes. (Figs. 10-19: scale bars represent $10 \mu \mathrm{m}$; Figs. 20-21: scale bars represent $20 \mu \mathrm{m}$ ). 

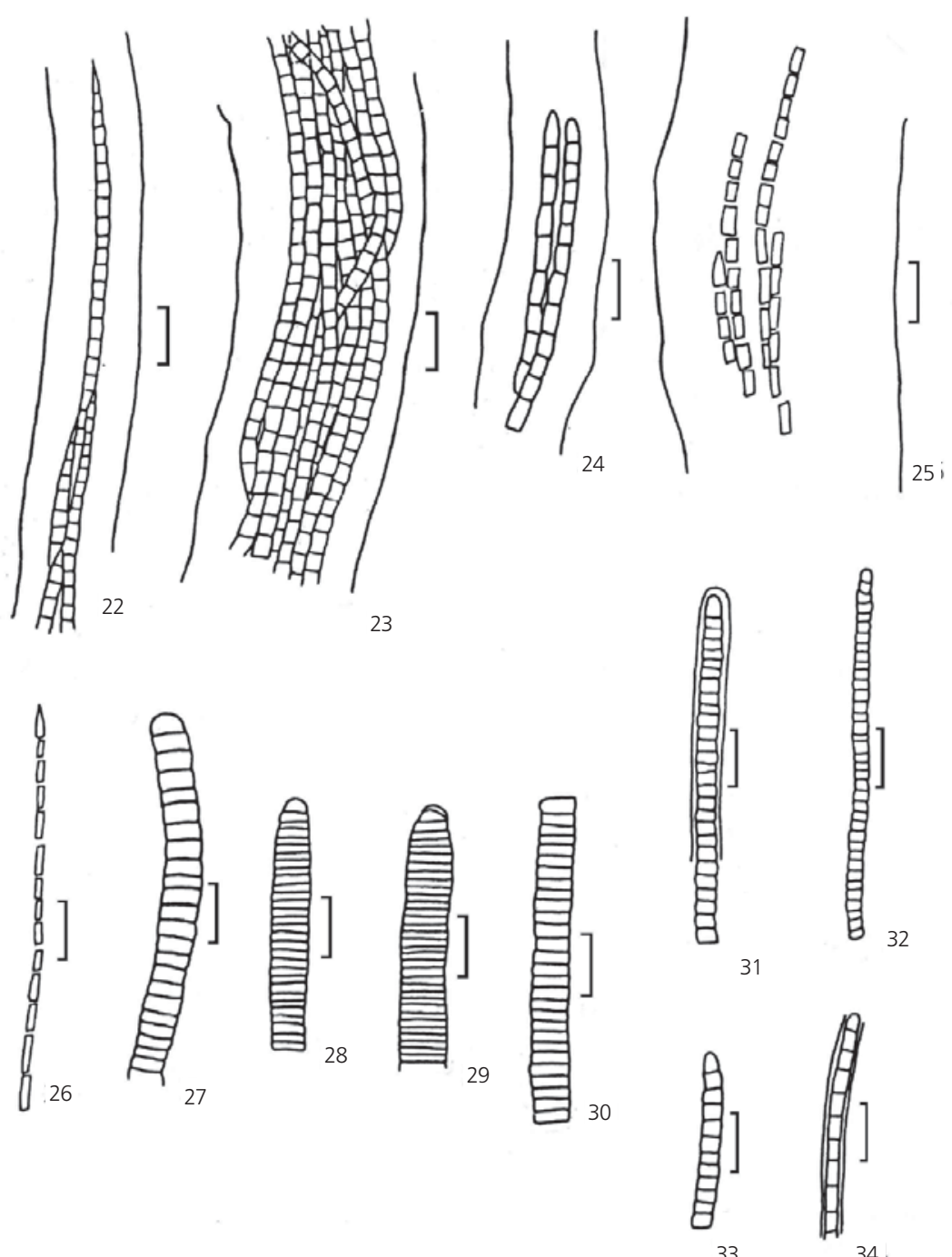

Fig. 22-24 - Microcoleus chtonoplastes Gomont. Fig. 22 - Acuminated apex; 23 - Many trichomes in a wide sheath; $24-$ Apical cell conic. Fig. 25-26 - Microcoleus tenerrimus Gomont. Fig. 25 - Trichomes in a wide sheath; 26 - Apical cell acute. Fig. 27-30 - Phormidium corallinae Gomont. Fig. 27 - General aspect; 28 - Apical cell conical; 29 - Apical cell with thickened outer membrane. Fig. 31-33 - Leptolyngbya cf. fragilis (Gomont) Anagnostidis \& Komárek. Fig. 31 Trichome with a hialine sheath; 32 - Trichome general aspect; 33 - Rounded apical cell. Fig. 34 - Leptolyngbya crosbyana (Tilden) Anagnostidis \& Komárek. (Scale bars represent $10 \mu \mathrm{m}$.)

\section{CONCLUSIONS}

A total of 15 species of algae growing on the trunks and roots of Rhizophora mangle L. were examined. These species were distributed within the families, as follows: Synechococcaceae (2), Chroococcaceae (1), Hyellaceae (1), Xenococcaceae (1), Oscillatoriaceae (1), Scytonemataceae (2), Phormidiaceae (5) and Pseudanabaenaceae (2).
Among the species identified, Leptolyngbya crosbyanum Tilden is reported for the first time for Brazil, and the other fourteen (14) species are cited for the first time for Maranhão. The species occurring in most of the samples were Scytonema insulare Sant'Anna, Scytonema arcangelii Bornet et Flahault, Microcoleus chthonoplastes Thuret ex Gomon, Leibleinia pellucida (Umezaki) Branco, SantAnna, Azevedo \& Sormus e Pleurocapsa sp. 
Acknowledgments - We wish to thank Dr. Célia Leite Sant' Anna, for helping with the taxonomic identification, Dr. Maria do Socorro Rodrigues Ibañez for reviewing the abstract and Dr. Cláudio Urbano Pinheiro for reviewing all of paper, and the National Technological Development Council (Conselho Nacional de Desenvolvimento Científico e Tecnológico - CNPq) for granting the first author a scholarship through the Federal University of Maranhão.

\section{REFERENCES}

ANAGNOSTIDIS, K. \& KOMÁREK, J., 1988, Modern approach to the classification system of cyanophytes. 3 Oscillatoriales. Algological Studies, 50-53: 327-472.

AZEVEDO, M. T. P. \& SANT'ANNA, C. L., 1993, New taxa of Oscillatoriaceae (Cyanophyceae) from São Paulo State, Brazil. Cryptogamic Botany, 3: 207-212.

BRANCO, L. H. Z., 1991, Cyanophyceae de comunidades bentônicas do Manguezal da Ilha do Cardoso, Município de Cananéia, SP. Dissertação de Mestrado, Universidade Estadual Paulista, Rio Claro, 152p.

BRANCO, L. H. Z., SANT'ANNA, C. L., AZEVEDO, M. T. P. \& SORMUS, L., 1996, Cyanophyte flora from Cardoso Island mangroves, São Paulo State, Brazil. 1. Chroococcales. Algological Studies, 80: 99-111.

BRANCO, L. H. Z., SANT'ANNA, C. L., AZEVEDO, M. T. P. \& SORMUS, L., 1997, Cyanophyte flora from Cardoso Island mangroves, São Paulo State, Brazil. 2. Oscillatoriales. Algological Studies, 84: 39-52.

CARMO, T. M. S., 1987, Os manguezais ao norte de Vitória, Espírito Santo. p. 180. In: Anais do Simpósio Ecossistemas da Costa Sul e Sudeste Brasileira. Vol. 1, Academia de Ciências do Estado de São Paulo (São Paulo, SP).

COUTINHO, R., 1982, Taxonomia, distribuição, crescimento sazonal, reprodução e biomassa das algas bentônicas no estuário da Lagoa dos Patos (RS). Dissertação de Mestrado, Fundação Universidade do Rio Grande, Porto Alegre, 232p.

CRONBERG, G., 1978, The Lago Paranoá Restoration Project: Phytoplankton ecology and taxonomy. Project PAHO/WHO 77/WT/BRA/2341/04, pp. 5-39.

CUTRIM, M. V. J., 1998, Distribuição espaço-temporal de macroalgas em troncos e pneumatóforos de Avicennia germinans (L.) Stearn em duas áreas de mangues da Ilha de São Luís, MA, Brasil. Tese de Doutorado, Universidade de São Paulo, São Paulo, 158p.

DAMÁZIO, E., 1979/1980a, Contribuição ao conhecimento da vegetação dos manguezais da Ilha de São Luís, MA. Parte I. Boletim do Laboratório de Hidrobiologia, 3(1): 9-12.

DAMÁZIO, E., 1979/1980b, Contribuição ao conhecimento da vegetação dos manguezais da Ilha de São Luís, MA. Parte II. Boletim do Laboratório de Hidrobiologia, 3(1): 57-76.
DAMÁZIO, E. \& SANTOS, M. C. F. V., 1985, Mapeamento do médio litoral com mangue do sul da Ilha de São Luís, MA. Boletim do Laboratório de Hidrobiologia, pp. 111-121.

DAMÁZIO, E. \& SANTOS, M. C. F. V., 1990, Mapeamento do médio litoral com mangue do sul da Ilha de São Luís, MA. pp. 11-121. Papers of the III Brazilian Meeting on Coastal Management, Anais do III Encontro Brasileiro sobre Gerenciamento Costeiro.

DESIKACHARY, T. V., 1959, Cyanophyta. Indian Council of Agricultural Research, New Delhi, 686p.

DOR, I., 1984, Epiphytic blue-green algae (Cyanobacteria) of the Sinai Mangal: consideration on vertical zonation and morphological adaptation. Boston, F.D. Por and I. Dor eds. Dr. W. Junk. The Hague, pp. 35-54.

DROUET, F., 1937, The Brazilian Myxophyceae. I. American Journal of Botany, 24: 598-608.

FERREIRA-CORREIA, M. M., 1986, Rodoficeas marinhas bentônicas do litoral oriental do Estado do Maranhão. Publicação da Pró-reitoria de Pesquisa e Pós-graduação. Coleção Ciências Biológicas. Série Botânica, 1, 253p.

FERREIRA-CORREIA, M. M. \& BRANDÃO, M. D. S., 1974, Flora ficológica marinha da Ilha de São Luís (Estado do Maranhão, Brasil). I Chlorophyta. Arquivo Ciências do Mar, 14(2): 67-80.

FERREIRA-CORREIA, M. M., LOPES, M. J. S. \& BRANDÃO, M. D. S., 1977, Levantamento das algas marinhas bentônicas da Ilha de São Luís (Estado do Maranhão, Brasil). Boletim do Laboratório de Hidrobiologia, 1(1): 23-46.

FRANCESCHINI, I., 1991, Algues d'eau douce de Porto Alegre (Brésil): taxinomia, composition floristique et peuplements. Univ. Paris (Thesis), Paris, 249p.

FROIS-ABREU, S., 1939, Observações sobre a Guiana Maranhense. Revista Brasileira de Geografia, 1/4: 26-54.

JOLY, A. B., 1951, Contribuição para o conhecimento da flora algológica do Estado do Paraná. Boletim do Instituto Paulista Oceanográfico, 2(1): 125-138.

JOLY, A. B., 1957, Contribuição ao conhecimento da flora ficológica marinha da Baía de Santos e arredores. Boletim da Faculdade de Filosofia e Ciências da Universidade de São Paulo, Série Botânica, 14: 1-199.

KOMÁREK, J. \& ANAGNOSTIDIS, K., 1989, Modern approach to the classification system of cyanophytes, 4: Nostocales. Algological Studies, 56: 247-345.

KOMÁREK, J. \& ANAGNOSTIDIS, K., 1999, Cyanoprokaryota I. Teil Chroococcales. In: H. Ettl, G. Gärtner, H. Heynig \& D. Mollenhauer (eds.), Süsswasserflora von Mitteleuropa, 19(1): 1-548, G. Fischer, Jena.

LAMBERT, G., STEINKE, T. D. \& NAIDOO, Y., 1989, Algae associated with mangroves in southern African estuaries: Cyanophyceae. South African Journal of Botany, 55: 476-491. 
MOCHEL, F. R., RODRIGUES, M. S., CUTRIM, M. V. J., FERREIRA-CORREIA, M. M., AZEVEDO, A. C. G., PESSOA, C. R. D., PACHÊCO, C. M., MAIA, D. C., IBAÑEZ, M. O. A., PWISECK, A. M. B., COSTA, C. F. M., SILVA, L. M., SILVEIRA, P. C. \& OLIVEIRA, V. M., Mangrove degradation in southwest São Luis Island, Maranhão, Northern Brazil: natural processes, human impact and consequence to local population. Unesco/SCI (in press).

MÖEBIUS, M., 1889, Bearbeitung der Van H. Schenk in Brasilien Gesammlten Algen. Hedwigia, 28(5): 309-347.

MORA, L. M. C., 1990, Chroococcales (Cyanophyceae) do Estado de Pernambuco, Brasil. II. Synechocystis, Synechococcus, Cyanothece, Merismopedia, Aphanothece, Gloeocapsa, Chroococcus, Gloeothece, Gomphosphaeria e Johannesbaptistia. pp. 139-169. In: Anais do IV Encontro Brasileiro de Plâncton.

MOURA, A. N., 1991, Estudo quali-quantitativo das algas perifíticas dos estuários dos rios Paripe e Igarassu Itamaracá, PE, Brasil. Dissertação de Mestrado, Universidade Federal de Pernambuco, Recife, 163p.

NEVES, M. H. B., 1983, Flora ficológica da lagoa hipersalina de Araruama (Estado do Rio de Janeiro-Brasil), 2: Cyanophyceae. Publicação do Instituto de Pesquisas da Marinha, 149p.

NEVES, M. H. B., 1988, Etude des Cyanophycées marines de la région de Cabo Frio (Rio de Janeiro, Brésil). Taxonomie et essai d'interprétation écologique. Tese de Doutorado, Universidade de Paris, Paris, 155p.

NEVES, M. H. B., 1990, As cianofíceas das salinas de Cabo Frio-Brasil. Acta Biológica Leopoldensia, 12(1): 99-123.

NEVES, M. H. B., 1991, Estudo das cianofíceas marinhas bentônicas da Região de Cabo Frio (Rio de Janeiro, Brasil) I-Chroococcales. Hoehnea, 18(1): 191-204.

NEVES, M. H. B., 1992, Estudo das cianofíceas marinhas da Região de Cabo Frio (Rio de Janeiro, Brasil) IIHormogonae. Rev. Brasil. Biol., 52(4): 641-659.

NEVES, M. H. B. \& TRIBUZI, D., 1992, Les Cyanophycées de la mangrove de la "Ponta do Pai Vitório" de la região de Cabo Frio (RJ, Brésil). Acta Biologica Leopoldensia, 14(2): $29-52$

OLIVEIRA, E. C., 1984, Brazilian mangal vegetation with special emphasis on the seaweeds. In: Hydrobiology of the Mangal. Por and I. Dor eds. Dr. W. Junk. The Hague, Boston, F. D., pp. 55-66.

SANT'ANNA, C. L., 1984, Flora de Cyanophyceae associada a briófitas, Município de Campina Verde, MG Rickia, 11: 129-142.

SANT'ANNA, C. L., 1988, Scytonemataceae (Cyanophyceae) from the State of São Paulo, southern Brazil. Nova Hedwigia, 46(3-4): 519-539.

SANT'ANNA, C. L., 1995, Cyanophyceae marinhas bentônicas do Parque Estadual da Ilha do Cardoso, SP, Brasil. Hoehnea, 22(1/2): 197-216.
SANT'ANNA, C. L., BICUDO, R. M. T. \& PEREIRA, H. A. S. L., 1983, Nostocophyceae (Cyanophyceae) do Parque Estadual da Ilha do Cardoso, Estado de São Paulo, Brasil. Rickia, 10: 1-27.

SANT'ANNA, C. L., CORDEIRO-MARINO, M., BRAGA, M. R. A. \& GUIMARÃES, S. M. P. B., 1985, Cianofíceas marinhas bentônicas das praias de Peruíbe e dos Sonhos, Município de Itanhaém, SP, Brasil,1. Rickia, 12: 89-112.

SANT'ANNA, C. L., SILVA, S. M. F. \& BRANCO, L. H. Z., 1991, Cyanophyceae da "Gruta-que-Chora", Município de Ubatuba, SP, Brasil. Hoenhea, 18(2): 75-97.

SANT'ANNA, C. L., AZEVEDO, M. T. P., BRANCO, L. Z., BRAGA, M. R. A., CORDEIRO-MARINO, M. \& GUIMARÃES, S. M. P. B., 1995, Cianofíceas marinhas bentônicas das Praias de Peruíbe e dos Sonhos, Município de Itanhaém, SP, III. Rev. Brasil. Biol., 55(3): 389-407.

SANT'ANNA, C. L. \& SIMONETTI, C., 1992, Cianofíceas marinhas bentônicas das Praias de Peruíbe e dos Sonhos, Município de Itanhaém, SP, II: espécies epilíticas e epizóicas. Rev. Brasil. Biol., 52(3): 515-528.

SANTOS, M. C. F. V., 1986, Considerações sobre a ocorrência de Rhizophora harrisonni Leechann e Rhizophora racemosa G. F. V. M. Meyer, no litoral do Estado do Maranhão, Brasil. Boletim Laboratório de Hidrobiologia, 7: 71-91.

SANTOS, M. C. F. C. \& DAMÁZIO, E., 1985, Um sistema de classificação para médio litoral com mangues. pp. 131-139. In: Anais do III Encontro Brasileiro de Gerenciamento Costeiro, Fortaleza, CE.

SANTOS, M. C. F. C., 1989, Strutural patterns of hyperhalinity stressed mangrove forests in the state of Maranhao, Northern Brazil. Masters Thesis, Department of Environmental Sciences, Colorado School of Mines, Golden, Colorado, 264p.

SANTOS, M. C. F. C., 1997, Intertidal salinity buildup and salt flat development in temperate salt marshes: a case study of the salt flats at the Virginia Barrier Islands. Dissertation (Doctor of Philosophy), Graduate Faculty of the University of Virginia, Virginia, 226p.

SENNA, P. A. C., 1992, Estudo das Nostocophyceae (Cyanophyceae) do Distrito Federal: Lagoas Joaquim Medeiros e dos Carás, 1. Rev. Brasil. Biol., 52(2): 259274

WERNER, V. R., 1984, Cyanophyceae (Nostocophyceae) planctônicas da Lagoa de Tramandaí e da Lagoa do Armazém, Rio Grande do Sul, Brasil: contribuição à taxonomia. Dissertação de Mestrado, Universidade Federal do Rio Grande do Sul, Rio Grande do Sul, 118p.

WERNER, V. R. \& ROSA, Z. M., 1992, Cyanophyceae da Estação Ecológica do Taim, Rio Grande do Sul, Brasil. Rev. Brasil. Biol., 52(3): 481-502. 